\title{
Research and Management Mechanism of Special Test Instrument Based on Knowledge Sharing and Value Sharing
}

\author{
Wei-Ming ZHAO, Lei LIANG, Li-Jie ZHANG \\ China Aerodynamics Research and Development Centre, Mianyang, China, 621000
}

Keywords: Knowledge sharing, Value sharing, Special test instrument, Research and management mechanism.

\begin{abstract}
Special test instrument used for aerodynamics research, especially instrument and equipment used for the field of flow visualization, possesses characteristics such as precious value and high technical content. Through analysis of current status of research and management of special test instrument, it brings in concepts of knowledge sharing and value sharing and probes into research and development and management mechanism of special test instrument so as to realize optimization of resource.
\end{abstract}

\section{Introduction}

Aerodynamics is to study on mechanical characteristics, gas movement law and accompanying physical and chemical change situation of flying machine or other objects when conducting relative motion with air or other gases. It is the important foundation for development of flying machine. Quality of aerodynamic test data is codetermined by multiple factors such as wind tunnel installation, test method, test equipment and technology, while test equipment and technology play an important role. Particularly research on flow phenomenon and law often has to seek help from advanced flow test technology, since it involves special test instrument with a large quantity, precious value and high technical content. Many of them require being developed on their own or introduced from foreign countries, thus requiring long-term and a mass of technology accumulation and in-depth research. Ministry of Science and Technology and Natural Foundation also treat development of instrument as major strategy to carry out.

To further improve level and efficiency of research and management of special test instrument, it introduces concept of knowledge sharing and value sharing into research and management mechanism of special test instrument.

\section{Basic concept of knowledge sharing and value sharing}

Aerodynamics is to study on mechanical characteristics, gas movement law and accompanying physical and chemical change situation of flying machine or other objects when conducting relative motion with air or other gases. It is the important foundation for development of flying machine. Quality of aerodynamic test data is codetermined by multiple factors such as wind tunnel installation, test method, test equipment and technology, while test equipment and technology play an important role. Particularly research on flow phenomenon and law often has to seek help from advanced flow test technology, since it involves special test instrument with a large quantity, precious value and high technical content. Many of them require being developed on their own or introduced from foreign countries, thus requiring long-term and a mass of technology accumulation and in-depth research. Ministry of Science and Technology and Natural Foundation also treat development of instrument as major strategy to carry out.

To further improve level and efficiency of research and management of special test instrument, it introduces concept of knowledge sharing and value sharing into research and management mechanism of special test instrument.

\section{Evolution of knowledge sharing}

Foreign and domestic scholars put forward multiple views on connotation of knowledge sharing based on different angles. According to starting point of the study, scholars roughly summarizes four viewing angles: information communication/ information flow angle, organizational learning angle, market angle and system angle [1]. 
In the process of knowledge sharing, effective sharing of knowledge can only be realized through mutual existence and interaction of relevant factors. Environmental factor of knowledge sharing is to create reasonable objective environment for knowledge sharing and offer environment-related protection for knowledge sharing; knowledge sharing object (knowledge) is the content of knowledge sharing, and sharing and communication of knowledge have formed knowledge flow of organization; knowledge sharing subject is the participator in knowledge sharing and the power source of knowledge sharing; knowledge sharing means is the carrier factor that supports smooth flow of knowledge and the technological foundation for implementation of knowledge sharing. Evolution of knowledge sharing can be described as: with certain environmental support, knowledge is transmitted and mutually transformed between individuals and between individual and organization through certain means .

\section{Platform of knowledge sharing}

From the perspective of information communication/ flow, knowledge sharing is the process of knowledge transmitting between individuals, namely the process of communication between individuals . Explicit knowledge means being acquired and easily learned by people through methods such as oral instruction, textbooks and reference data. Implicit knowledge is mainly composed of two elements, cognitive and technical skills. Cognitive element is deep hidden in mind of technicians, such as shared vision, organizational culture, value, insight, sentiment and loyalty to the organization; technical element refers to informal the so-called "knack" that is difficult to master, craftsmanship, technique and experience derived from practice. Knowledge sharing, especially implicit knowledge sharing requires knowledge sharing platform. Lai Huirong puts forward that it requires concentrating efforts in constructing communication platform, establishing learning mechanism, incentive mechanism and shaping excellent library organizational culture to successfully share implicit knowledge . Song Yan has constructed knowledge sharing support mechanism of virtual academic team from confidence mechanism, situational mechanism and communication mechanism . Cong Haitao and Tang Yuanhu have discussed implicit knowledge transfer, design method of sharing incentive mechanism and proposed implicit knowledge transfer, sharing incentive mechanism design model [2].

\section{Value sharing}

Internal meaning of value sharing is to conduct decision-making on law of value based on value driving. Two scholars Wang Huacheng and Yin Meiqun made more complete elaboration of value system of value chain based on proposed value net . They defined influencing factors of value as mutual relationship among customer value, core competence and value chain nodes among organizations with a view to analysis of creation system of value chain. In these three elements, customer value holds the core location and through value driving and sharing, core competence and mutual relationship are finally reflected comprehensively through customer value. All effects of value driving and sharing elements should eventually be implemented on talents, personal effort and cooperative effort of managerial staff and managerial staff at all levels, R\&D personnel and general personnel.

\section{Some problems in existing research and management mechanism of special test instrument}

\section{Evolution of knowledge sharing not deep enough}

Special test instrument changes with each passing day and new generation emerges endlessly, whose adopted forms of development and use now mainly are "outsourcing" and direct sourcing. In the development process of instrument, user and contractor have difficulty in knowledge sharing, leading to deviation of expectation and sluggishness of progress; in the transition process of instrument, it lays emphasis on transition of entity and has lost sight of effective knowledge sharing evolution, only knowing operation and simple maintenance of instrument, which has given rise to insufficient analysis and grasping of more in-depth working principle and difficulty in use after delivery as well as bringing bad influence on carrying out work to a certain extent. 


\section{Incomplete knowledge sharing platform}

To facilitate management, valuable special test instrument often adopts centralized management mode to be operated and maintained by specially-assigned person. Due to lacking of a complete knowledge sharing platform, it will easily cause disjointing. Equipment personnel only knows operation of the equipment without knowing data analysis and hasn't been involved in taking advantage of equipment to solve problem; analyst only makes data analysis and application without concerning about how data is obtained and what the working principle of equipment is. They only know it's so, but don't know why it's so, which is not conducive to accurate judgment of analysis result. Since implicit knowledge has difficulty in sharing, when new technician is unable to effectively share previous mistakes as using special test instrument, which seriously affects efficiency, or even interrupts research process under compulsion because of small mistakes.

\section{Value sharing has not been well played}

In the practice, special test instrument is generally coordinated and used for free through mandatory plan or given symbolic economic compensation. In the meantime, it prefers to economic value while ignores academic value and spiritual value on value orientation without giving full play of value driving, leading to special test instrument's instrument capacity hard to be given full play in research and management.

It introduces concept of value sharing into research and management mechanism of special test instrument, namely taking value orientation as lever to improve performance of research and management of special test instrument and realize the objective of multi-win.

\section{Introduction of knowledge sharing and value sharing}

Through analysis of current status of research and management mechanism of special test instrument, it found that failure to apply concept of knowledge sharing and value sharing well is a factor that restricts development and promotion capacity of existing special test instrument.

\section{Strategy to accelerate evolution of knowledge sharing}

To accelerate knowledge sharing and evolution of this organization and other organizations, it can start with following several aspects:

First is to establish incentive and restrict mechanism. Incentive and restrict mechanism can promote knowledge sharing. Since every organization is independent legal entity and aims to pursue self benefit maximization, it requires increasing revenue of knowledge sharing and cost of knowledge non-sharing. If the power of knowledge sharing comes from revenue generated from act of knowledge sharing. Multi-faceted revenue here can either be revenue in materials or revenue in spirit.

Second is to strengthen knowledge coordinated and innovative capacity and absorption and transformation capacity. In bilateral cooperation of knowledge sharing, the stronger the coordinated and innovative capacity and self-absorption and transformation capacity are, the greater the coordinated revenue it generates, the more abundant knowledge achievement it achieves, the greater revenue two parties gains, thus they are more willing to take the action of knowledge sharing.

Finally is to establish consortium cooperative mechanism. It has to consider organization with strong knowledge learning and innovative capacity, high willingness of knowledge sharing and good reputation so as to build up long-term and stable cooperative relationship. The longer and more enduring the cooperation is, the high credibility of members is and future expectation can be paid more attention to avoid short-term act. In general, strong connection of organization is conducive to knowledge sharing.

\section{Construct virtual platform of knowledge sharing}

Construct virtual platform of knowledge sharing is to enable technician who requires acquiring shared knowledge to know where to find knowledge as required as well as to help personnel who owns knowledge to share knowledge with others, especially implicit knowledge. This platform can be a training system, a rotation work pattern, a document management mode and a communication system, etc., and of course, it can be completely a combination of above one and several manners. 
Information technology has integrated into various professional fields and plays an increasingly important role, which not only solves problem of the equipment, but also plays an important integration role in cross-instrument and cross-system, thus greatly improving production efficiency. On the platform of knowledge sharing, it still requires taking advantage of existing network means to set up virtual academic platform of knowledge sharing and strengthen professional construction force. To construct universal trust-oriented virtual platform that encourages knowledge sharing, synergetic effect and superposition effect can also be increased with impact of information network so as to realize information integration and make the knowledge sharing develop towards standardized and intellectualized directions.

\section{Introduction of value sharing}

In the aspect of organizational structure, traditional pyramid bureaucratic organization setting also causes spatial and hierarchical obstacles to various managerial hierarchies. One of the most effective ways of shared value is to utilize the level of value driving to construct compressed organizational structure or project unit, which can better give full play of technician's dynamic role and improving efficiency of special test instrument.

In the aspect of service object of work task, it adopts research and management strategy divided and ruled by different types of organizational systems. Organization focusing on equipment development can stimulate technician's enthusiasm driven by economic benefit; project (research) group centering upon pneumatic basic research can drive and give play to technician's master spirit in a manner of joining project (research) group; cooperation and exchanged-based academies can adopt forms of academic exchange and academic training to drive technician to share knowledge with direction and channel.

\section{Conclusions}

Through introducing knowledge sharing and value sharing into research and management mechanism of special test instrument: strong connection of organizations' evolution of knowledge sharing and construction of virtual platform for knowledge sharing and mode of value sharing driven by non-economic value can promote test data's abilities to conduct comprehensive production, analysis, evaluation and planning, thus realizing the objective of optimizing resource.

\section{References}

[1] Bartol K M,Srivastava A. Encouraging Knowledge Sharing:The Role of Organizational Reward Systems [J]. Journal of Leadership \& Organizational Studies, 2002 (1):64-76.

[2] Andrews KM,Delahaye BL. Influence on Knowledge Processes in Organizational Learning: The psychosocial filter [J]. Journal of Management Studies, 2000, 37:797-810. 